\title{
The Self-Assembling Process and Applications in Tissue Engineering
}

\author{
Jennifer K. Lee, ${ }^{1,3}$ Jarrett M. Link, ${ }^{1,3}$ Jerry C.Y. Hu, ${ }^{1}$ and Kyriacos A. Athanasiou ${ }^{1,2}$ \\ ${ }^{1}$ Department of Biomedical Engineering, University of California, Davis, California 95616 \\ ${ }^{2}$ Department of Orthopaedic Surgery, University of California, Davis, California 95616 \\ Correspondence: athanasiou@ucdavis.edu
}

Tissue engineering strives to create neotissues capable of restoring function. Scaffold-free technologies have emerged that can recapitulate native tissue function without the use of an exogenous scaffold. This review will survey, in particular, the self-assembling and self-organization processes as scaffold-free techniques. Characteristics and benefits of each process are described, and key examples of tissues created using these scaffold-free processes are examined to provide guidance for future tissue-engineering developments. We aim to explore the potential of self-assembly and self-organization scaffold-free approaches, detailing the recent progress in the in vitro tissue engineering of biomimetic tissues with these methods toward generating functional tissue replacements.

$\mathrm{T}_{\mathrm{p}}^{\mathrm{h}}$ he field of tissue engineering aims to recapitulate native tissue function toward replacing damaged or diseased tissues and organs. The tissue-engineering paradigm is traditionally composed of living cells, scaffolds, and signals. The scaffolds used in tissue engineering are highly diverse, ranging from synthetic to natural polymers and hydrogels to woven meshes. The emergence of scaffold-free processes-tissueengineering platforms that do not require the addition of an exogenous scaffold-have expanded the capabilities of the field. Scaffoldfree techniques have been successfully used in engineering musculoskeletal (DuRaine et al. 2015) as well as cardiovascular, metabolic, and corneal tissues (Athanasiou et al. 2013). Within scaffold-free approaches, two primary, thermo- dynamically driven modalities have been described: self-organization and self-assembly (Fig. 1) (Athanasiou et al. 2013).

In this review, a summary of the progress in tissue engineering will be covered. Although a wide variety of cell sources and stimuli can be applied in tissue engineering, the focus of this work will be related to scaffold-free approaches. Herein we focus specifically on the in vitro tissue-engineering techniques that generate biomimetic tissues (i.e., those that recapitulate native tissue). In vivo tissue engineering can also include the injection of cell suspensions and matrix-associated cells for continued development and maturation in an in vivo environment, but are not extensively covered here. Finally, the progress in engineering functional

${ }^{3}$ These authors contributed equally to this work.

Editor: Joseph P. Vacanti

Additional Perspectives on Tissue Engineering and Regenerative Medicine available at www.perspectivesinmedicine.org

Copyright (C) 2017 Cold Spring Harbor Laboratory Press; all rights reserved; doi: 10.1101/cshperspect.a025668

Cite this article as Cold Spring Harb Perspect Med 2017;7:a025668 
J.K. Lee et al.

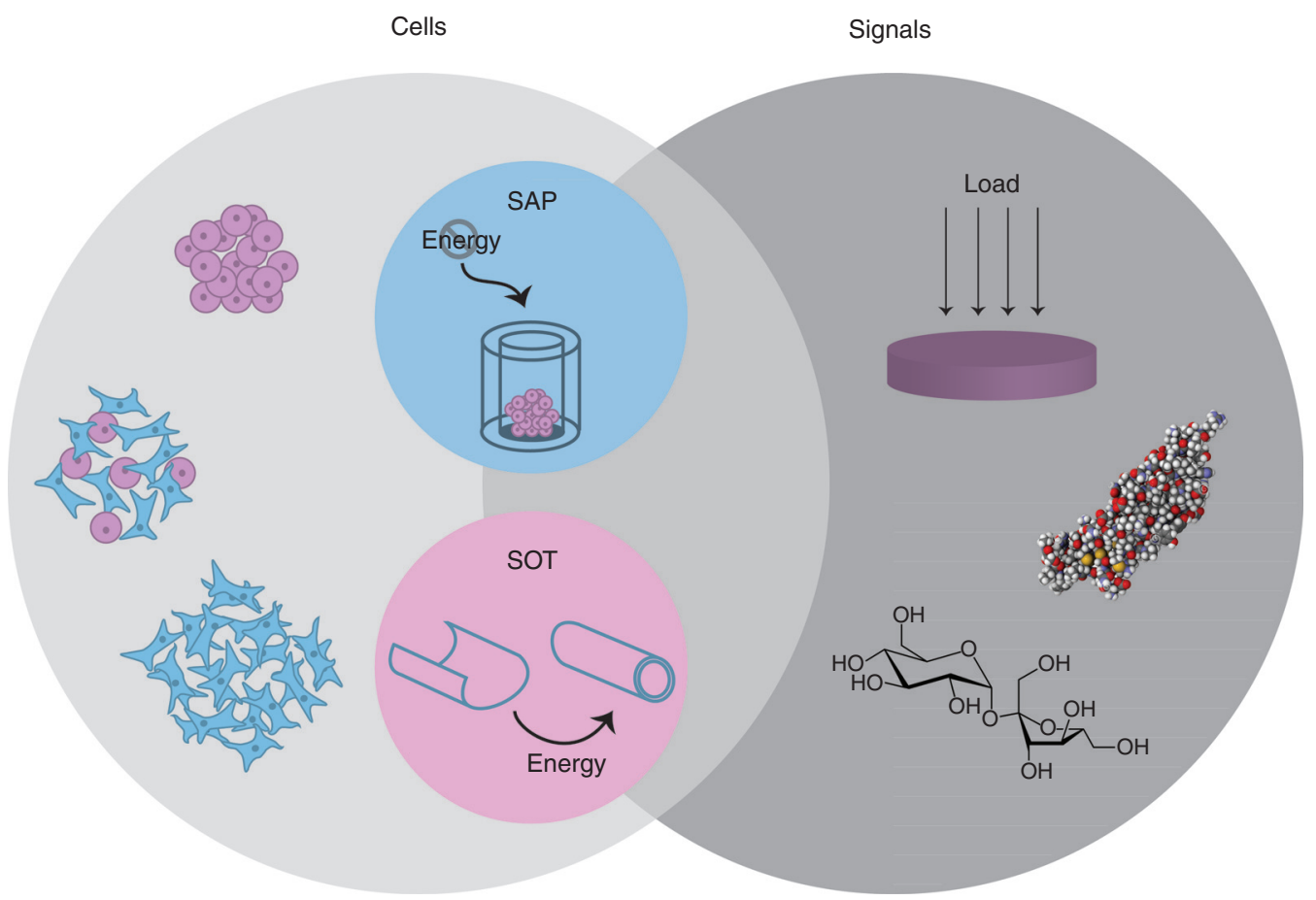

Figure 1. Scaffold-free tissue engineering. The tissue-engineering paradigm typically consists of cells, scaffolds, and signals. The benefits of scaffold-free approaches have motivated the use of only cells and signals. Depicted here are example modalities within this paradigm, using homogeneous or heterogeneous cell populations in concert with mechanical (e.g., compressive loading) and/or biochemical stimuli (e.g., transforming growth factor $\beta 1$ [TGF- $\beta 1]$ or sucrose) to enhance neotissue properties. Two distinct forms of scaffold-free tissue engineering exist, termed the self-assembling process (SAP) and the self-organization technique (SOT). Although self-organization requires the exogenous input of energy, self-assembly occurs in a closed system.

tissues with a particular emphasis on self-assembling and scaffold-free techniques to treat a wide range of diseases will be highlighted.

\section{TISSUE ENGINEERING}

Classical tissue-engineering approaches combine cells, biomaterials, and bioactive stimuli to generate robust implants capable of restoring the structure and function of tissues damaged by trauma, pathology, or age. Often referred to as the tissue-engineering "triad," this foundational concept of cells, scaffolds, and signals has informed strategies for numerous outcomes, such as bone regeneration following complex fractures or the development of vasculature in vitro to replace diseased vessels (Chaparro et al. 2015; Shimizu et al. 2015). Signifi- cant advances in the field have resulted from this paradigm. However, scaffold-free techniques have emerged, which may better apply to certain tissues in which cells may not require exogenous scaffolds. In this manner, biomimetic and functional tissues of clinically relevant dimensions may be created. Scaffold-free techniques may thus improve the likelihood for clinical translation, which remains the ultimate goal of the field.

\section{SELF-ASSEMBLING PROCESS}

One promising tissue-engineering technique, especially in cartilage tissue engineering, is the self-assembling process $(\mathrm{Hu}$ and Athanasiou 2006; Athanasiou et al. 2013). Without the influence of external energy, self-assembly mimics 
developmental events to generate functional cartilaginous tissue with characteristics reminiscent of native tissue (Fig. 2). Nonadherent culture substrates-typically agarose-support high-density chondrocyte seeding, prevent cell attachment, and encourage cell-cell interactions, facilitating the chondrogenic phenotype. Indeed, cell adhesion is up-regulated in chondrocytes (Ofek et al. 2008) during the initial phase of the self-assembling process, reminiscent of mesenchymal condensation during cartilage morphogenesis (Tavella et al. 1994; DeLise et al. 2000). For example, increased levels of $\mathrm{N}$-cadherin on the cell surface can minimize free energy, according to the differential adhesion hypothesis as described below. Critically, no external energy is provided to the system during self-assembly (i.e., it is a closed system). Since the development of the self-assembling process, substantial efforts toward understanding the mechanisms of action have been inves- tigated to refine the technique further. In particular, the differential adhesion and differential interfacial tension hypotheses have been used to describe the self-assembling process.

Informing the mechanism of the self-assembling process, the differential adhesion hypothesis posits that tissues minimize free energy via cell-cell binding. The type and number of adhesion proteins present on a cell surface give rise to cell-cell interactions. Correspondingly, a mass of cells behave analogously to a liquid and will minimize its surface tension, known as tissue surface tension. This tension will determine the sorting behavior of cells in a mixed population, as cells with higher surface tension will sort to the center, maximizing intercellular adhesion. Consequently, tissue surface tension will be minimized. Similarly, in the self-assembling process, the nonadherent substrate forces a homogeneous cell population to minimize free energy via cell-cell adherence, facilitated by in-
A

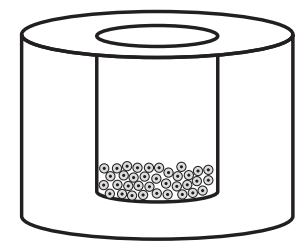

E

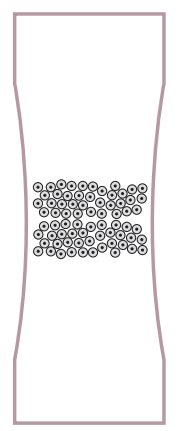

B

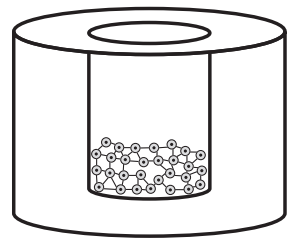

$\mathbf{F}$

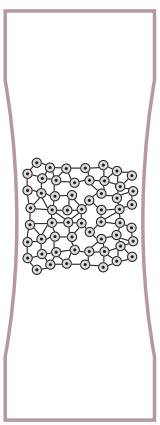

C

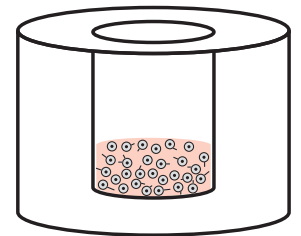

G

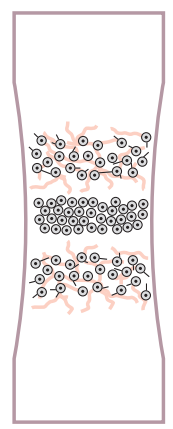

D

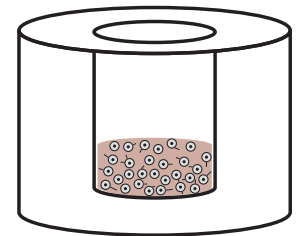

H

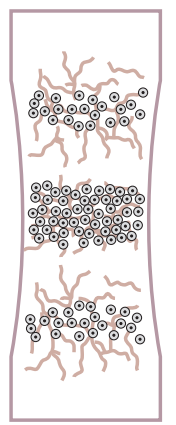

Figure 2. The phases of self-assembly and cartilage development. Self-assembling articular cartilage forms in a manner reminiscent of cartilage morphogenesis. In the first phase of self-assembly $(A)$, a high-density cell solution is seeded in a nonadherent well. During phase $2(B)$, minimization of free energy occurs as cells bind to one another via cell-adhesion receptors like $\mathrm{N}$-cadherin. In phase $3(C)$, extracellular matrix synthesis is up-regulated. Finally, the engineered tissue matures as distinct regions develop and native tissue-like functional properties are approached $(D)$. Similarly, the process of long bone formation is mediated first by mesenchymal condensation $(E)$. Robust matrix deposition begins as cells differentiate $(F)$, following chemotactic agents to elongate the bone in opposite directions $(G)$. Over time, the core forms a site for vascularization to become bone $(H)$. 
J.K. Lee et al.

creased levels of $\mathrm{N}$-cadherin and other adhesion molecules (Ofek et al. 2008; Raghothaman et al. 2014). The development of this continuous aggregate is critical to neotissue development, reflects the process of mesenchymal condensation, and can potentially drive chondrogenic gene expression (Ofek et al. 2008; Raghothaman et al. 2014). The mechanism of self-assembly can thus be partly explained by the differential adhesion of surface-bound molecules.

Another mechanism that may contribute to the self-assembling process is differential interfacial tension. Cortical cell tension, driven by contractility of the actin cytoskeleton and cell surface tension, has been implicated in cell sorting (Brodland 2002; Krieg et al. 2008; Manning et al. 2010). As in differential adhesion, the minimization of free energy drives the cellular behavior in the differential interfacial tension hypothesis, with cell sorting dictated by forces generated by the cell cytoskeleton and at the cell membrane. Specifically, cells generating similar tensions will tend to aggregate as compared with those showing different tensions. The differential adhesion and differential interfacial tension hypotheses may be related (Manning et al. 2010). Increased understanding of the relative contributions and/or interactions of these processes would help elucidate self-assembly mechanisms.

Drawing from knowledge of developmental biology, biomedical engineers can use the selfassembling process to drive cell sorting, gene expression, and tissue formation in a manner similar to morphogenesis. Our enhanced understanding of underlying mechanisms in self-assembly will drive the rational selection of agents that can positively modulate the formation of tissues with increased functional properties.

\section{OTHER SCAFFOLD-FREE TECHNIQUES}

Although promising, self-assembly is but one example of a variety of scaffold-free tissue-engineering methodologies that have gained traction and present unique advantages. For instance, scaffold-free systems do not produce synthetic degradation by-products, can maintain the rounded phenotype of cells such as chondrocytes, and do not require harsh processing chemicals involved in scaffold production (Huey et al. 2012). Many of these alternate techniques can be grouped within the self-organizing tissue-engineering paradigm, which is a distinct subset of scaffold-free tissue engineering that requires external energy input into the system for tissue formation (Fig. 1). Moreover, these varied techniques are able to form tissues with a range of dimensions (Fig. 3). Within this paradigm exist methods such as pellet culture, aggregate culture, and cell-sheet engineering.

Pellet culture is a fundamental method of scaffold-free tissue engineering. Requiring substantial external energy, pellet culture is mediated by centrifugation of cells inside a conical-shaped tube. Subsequently, cell pellets are cultured in medium specific to a certain tissue, driving cellular differentiation to achieve tissuespecific gene expression and extracellular matrix (ECM) deposition. Bone, liver, and cartilage tissues have all been formed in this manner (Ong et al. 2006; Gurkan et al. 2011; Diekman et al. 2012). Despite the ability to create tissues with relevant ECM components, pellet culture fails to meet many clinical translation criteria, such as robust mechanical properties and anatomically relevant geometries and dimensions. Thus, biomedical engineers interested in translational medicine typically focus on strategies other than pellet culture for use in in vitro biomimetic tissue engineering.

A variety of methods exist to generate cellular aggregates or spheroids, and these terms are often used interchangeably in the literature. Similar to pellet culture, aggregate culture maintains cells in a 3D environment and can be used to enhance tissue-specific gene and protein expression. Multiple methods are used to induce aggregate formation: hanging drop, round, or v-bottom well-plates, and rotational culture. In hanging-drop culture, cell suspensions are placed in droplets on the lid of a culture plate; after inverting, gravity assists cells in coalescing at the base of the drop, forming an aggregate. Similar to self-assembly, the well-plate method uses nonadherent roundor v-bottom plates to statically induce aggregate 


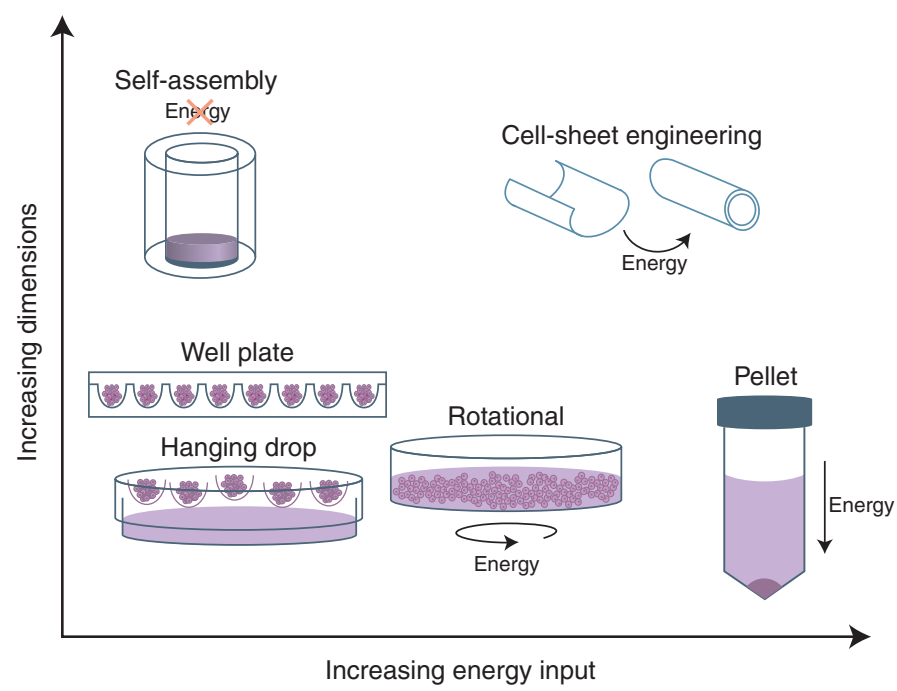

Figure 3. Energy usage and achievable dimensions in scaffold-free processes. Scaffold-free processes differ with respect to the energy required for tissue formation and the dimensions that can be achieved. Spheroid or aggregate formation based on the methods of hanging drop, round, or v-bottom well plates, or rotational culture requires minimal energy and forms aggregates of small dimensions. Pellet culture requires substantial energy in the form of centrifugation and similarly forms small-diameter cellular aggregates. Finally, self-assembly and larger self-organization techniques such as cell-sheet engineering are able to generate sizeable constructs of clinically relevant dimensions.

formation. Finally, by subjecting a cell population to rotational culture in the presence of tissue-specific growth factors, aggregation and differentiation is encouraged through cell-cell and cell-matrix interactions. Subsequently, relevant ECM proteins are synthesized and neotissue begins to form. Although these methods are able to form aggregates that can serve as important tools in understanding mechanisms of differentiation and phenotypic maintenance, they may not be suitable for in vitro biomimetic tissue engineering.

Aggregate or spheroid culture, like pellet culture, produces small-diameter cellular aggregates and may not be a feasible approach to engineer mechanically functional tissues when used alone, likely because of the limited number of cells in each aggregate. If the approach is part of a larger tissue-engineering effort (Murphy et al. 2013a), however, then it can be used to engineer anatomically relevant tissues. For instance, aggregate culture can encourage both differentiation of stem cells and redifferentiation of passaged primary cells, followed by their application in other tissue-engineering methods, such as self-assembly, to create larger constructs (Murphy et al. 2015). Bioprinting has emerged as a method to use directly these small diameter aggregates as "bioink," which are fed through a small nozzle and deposited in specific locations during $3 \mathrm{D}$ printing. Additionally, with fusion of multiple spheroids, larger continuous constructs can be generated. Indeed, application of compression to mesenchymal stem cell (MSC) aggregates within a mold has been able to generate large and continuous cartilage constructs (Bhumiratana et al. 2014). Thus, although the tissues formed by both pellet and aggregate/spheroid culture may not reach clinically relevant dimensions, these methods are important for the phenotypic maintenance of many cell types and can be integrated as part of a larger tissue-engineering process.

Cell-sheet engineering is a scaffold-free approach using external manipulations and thermal energy to form 3D tissues. Cells are cultured in monolayer on functionalized substrates or on a thermoresponsive polymer. In the case of 
J.K. Lee et al.

direct cellular attachment, cells are removed via enzymatic cleavage of their cell-matrix attachments to the surface; to avoid enzymatic detachment, cell scraping can also be used. The cell sheet can be draped over a mandrel to form, for example, a hollow vascular structure (L'Heureux et al. 1998). Alternatively, thermoresponsive polymers have been developed in which, subject to a change in temperature, the polymer changes conformation and induces detachment of the cell layer (Nishida et al. 2004a). The polymer method avoids the use of mechanical or enzymatic cell detachment to preserve the cell-matrix-binding interactions. Further, fusion of multiple cell-sheet layers can be used to generate tissues of greater thickness to recapitulate the zonal architecture of target tissues (Shimizu et al. 2006). As compared with pellet and aggregate culture techniques, cell-sheet engineering can form substantially larger structures.

\section{ADVANTAGES OF SCAFFOLD-FREE TECHNIQUES}

The process of engineering tissues using scaffold-free techniques shows distinct advantages. For instance, scaffold-free methods may promote native tissue integration, facilitate enhanced matrix deposition and, thus, more direct mechanotransduction, and avoid the release of harmful by-products. In the case of self-assembled articular cartilage, the neotissue has an abundance of cells at the construct edge, which likely encourages tissue growth into native cartilage and promotes integration (Athens et al. 2013). Direct cell-ECM interactions in self-assembled cartilage prevent cells from experiencing stress shielding, which has been shown to impede matrix production and remodeling (Hu and Athanasiou 2006). Scaffold-free approaches can avoid issues of cytotoxicity caused by the harsh processing conditions-particulate-leaching polymerizing chemicals and plasticizers, for example-required for manufacturing of some biomaterials (Vunjak-Novakovic et al. 1999). Without the use of foreign materials, scaffold-free processes can reduce the likelihood of a foreign material-associated immune re- sponse; this response is known to limit the durability of the implanted construct and potentially compromise the health of the patient (Avula et al. 2014). Biocompatibility issues, assuming a cell source is chosen appropriately, are mitigated in the scaffold-free paradigm because synthetic materials are avoided. Scaffold-free techniques thus possess several advantages for use in tissue-engineering strategies.

Yet, there are limitations associated with the scaffold-free paradigm. If scaffold-free techniques cannot match native tissue functional properties at implantation, clinical translation is complicated. For instance, if engineered cartilage with inferior mechanical properties were placed into a femoral focal defect, stress concentrations could develop within the engineered cartilage and at the native-engineered tissue interface. Especially in the case of load-bearing tissues, mismatch in functional properties could result in destruction of the implant if not appropriately unloaded postsurgery. Physicians may have to devise rehabilitation regimens specific to scaffold-free constructs to improve the clinical viability of these approaches. To ensure clinical success of scaffold-free approaches, application of biomimetic stimuli (Huey et al. 2012; Athanasiou et al. 2015; Makris et al. 2015) is crucial to driving the development of functionally relevant neotissues.

Scaffold-free approaches often require a high cell-seeding density, which brings into question the issue of cell sourcing. It is important to note that certain cell types are anchorage-dependent and require the presence of an exogenous scaffold at seeding; these cells may not be suitable for a scaffold-free approach. Primary autologous cell-harvesting techniques often do not meet cell number requirements and can be associated with donor site morbidity (Amini et al. 2012). Passaged primary autologous cells are available in higher quantities, but may be limited by expansion potential and do not address the issue of donor site morbidity. Furthermore, passaged primary cells may experience epigenetic changes that affect gene expression (Darling and Athanasiou 2005), leading to an altered ECM profile and, potentially, reduced functional properties. Stem cells, 
such as MSCs and dermis-derived stem cells, represent an attractive cell source as they are more widely available and have shown the ability to differentiate into many different cell and tissue types. In many cases, however, stem cells do not fully differentiate into the target cell, which may affect the ultimate properties of generated neotissue. Moreover, differentiation protocols are often complex and may result in nonhomogeneous cell populations. Cell sourcing remains a critical issue in tissue engineering and must be solved to improve the prospects of clinical translation for scaffold-free approaches.

Tissue-specific design criteria must be considered when choosing a particular tissueengineering approach. In some cases, a selfassembly model may be ideal. For example, chondrocytes are particularly amenable to the self-assembling process: passaged rabbit cells treated with a combination of bioactive (i.e., transforming growth factor $\beta 1$ [TGF- $\beta 1$ ] and chondroitinase-ABC) and mechanical (i.e., hydrostatic pressure) stimuli can create tissues with clinically relevant dimensions with a tensile stiffness reaching 6.3 MPa (Arzi et al. 2015). On the other hand, a scaffold may be necessary for recapitulating the structure-function relationship in a large segmental defect of bone, as osteoblasts require a scaffold for survival. Researchers must continue to refine these techniques and further define native tissue structure and function to develop the most effective tissue-engineering strategies. We would like to emphasize the importance of biomimetic, functional tissue engineering, which will be discussed at length later in this article.

\section{SCAFFOLD-FREE TISSUE ENGINEERING IN THE CLINIC}

Although obstacles still exist, promisingly, a few scaffold-free processes have reached the clinic. Autologous chondrocyte implantation (ACI), although not an in vitro biomimetic tissue-engineering method, was established in 1994 as a clinical treatment for focal articular cartilage defects (Brittberg et al. 1994). Requiring two surgical steps-one to harvest tissue, the other to implant cells into a defect-ACI has been shown to be superior to other surgical techniques such as mosaicplasty (Bentley et al. 2012), although other studies have shown that $\mathrm{ACI}$ is no better functionally than other cheaper orthopedic procedures like microdrilling or microfracture (Tuan 2007; Lim et al. 2012). In ACI, the defect may be filled with reparative tissue synthesized by implanted articular chondrocytes; this fibrocartilaginous tissue shows functional properties inferior to native hyaline articular cartilage. Thus, ACI may lead to a limited repair response. Furthermore, this technique may be less effective in older patients, who are more likely to suffer from musculoskeletal maladies as a result of the reduced proliferative and regenerative capabilities of aged cells. However, the clinical development pathway for ACI can inform the translation of scaffold-free in vitro biomimetic tissue-engineering techniques.

In a biomimetic scaffold-free approach, cell-sheet-engineered vasculature for end-stage renal disease patients undergoing hemodialysis has shown promise in clinical trials (McAllister et al. 2009). Scaffold-free techniques for vascular tissue engineering have shown a higher propensity for achieving native tissue structure and can withstand higher burst pressures (L'Heureux et al. 2006). Additionally, removing the influence of a biomaterial reduces the chance of dangerous thrombosis formation and leukocyte activation. In this clinical trial, concerns related to unrolling of the cell sheet arose (McAllister et al. 2009). Reported cases of unrolling identify a critical shortcoming of cellsheet engineering for vascular tissue engineering; methods to ensure fusion of the cell sheet via improved nutrient transport may promote long-term closure (McAllister et al. 2009). If the issues of extended culture times and the potential for unrolling can be solved, in vitro cellsheet engineering for diseased vasculature may experience broad clinical use.

In a biomimetic tissue-engineering approach akin to self-assembly, ISTO Technologies in concert with Zimmer Biologics has produced scaffold-free neocartilage that successfully completed phase I/II trials and continues on the path of commercialization. The RevaFlex prod- 
J.K. Lee et al.

uct is generated from a high-density cell culture derived from juvenile donors; the investigators previously determined that chondrocytes derived from younger donors possessed enhanced neocartilage-generation potential (Adkisson et al. 2001). RevaFlex was implanted into 12 patients in a phase I/II clinical trial initiated in late 2006 (McCormick et al. 2013). In this clinical study, clinical efficacy was assessed via patient-reported outcome measures, magnetic resonance imaging (MRI), and elective secondlook arthroscopy and biopsy (McCormick et al. 2013). Of second-look arthroscopies (nine of 12 patients), RevaFlex reportedly resulted in " $26 \%$ of lesions show[ing] gross anatomical cartilage characteristics with adequate fill with promising histologic characteristics" (McCormick et al. 2013). Additionally, no immunological response to allograft neocartilage was found; these findings of a lack of graft rejection address many clinical concerns associated with using juvenile cells from an allogeneic source for graft production (Lu et al. 2005; McCormick et al. 2013). RevaFlex received Food and Drug Administration (FDA) approval for an investigational new drug (IND) application before clinical trials and is thus regulated as a biologic, rather than a device. Ultimately, the commercial success of RevaFlex paves the way in regulatory aspects of scaffold-free tissues-particularly in terms of cell sourcing and immune rejectionand will inform the clinical development of cartilage constructs generated from self-assembling or self-organization techniques.

Although translation of scaffold-free techniques to the clinic is limited, these advances illustrate the required regulatory path for clinical success. For instance, cell-based therapies like ACI may provide some insight into clinical translation of scaffold-free tissue-engineered therapies. Although these cell therapies do not fall into the scope of biomimetic in vitro tissue engineering, they are informative examples of clinical translation of cell-derived products. RevaFlex is an example of an in vitro engineered, biomimetic articular cartilage tissue replacement; the path to FDA approval of RevaFlex highlights the challenges of clinical translation of such a product. Given the similarities between the self-assembling process and the methods used to generate the RevaFlex graft, the RevaFlex pathway provides guidance for clinical translation of a self-assembled articular cartilage replacement. Although continued refinement of these scaffold-free tissue-engineering techniques is required, lessons from approved, clinically available cell-based and biomimetic tissue-engineered therapies should be noted.

\section{FUNCTIONAL TISSUE ENGINEERING}

Biomimetic tissue engineering aims to generate functional tissues in vitro to achieve certain properties of target tissues; these properties depend on the tissues' primary roles in vivo. The benchmarks for engineered tissues are derived from their native counterparts. For instance, musculoskeletal tissue engineers focus on forming tissues with appropriate mechanical strength and stiffness to function in the tissues' native load-bearing capacities. Hepatic tissue engineers focus on forming tissues able to express specific enzymes and proteins necessary to mimic liver function. Some of these commonly reported parameters are presented in Table 1. Scaffold-free tissue-engineering techniques aiming to recapitulate native tissue function should bear in mind these reported properties and strive to bring them closer to native tissue values. The following sections highlight recent progress in engineering musculoskeletal, cardiovascular, liver, and corneal tissues using scaffold-free biomimetic in vitro tissue engineering.

\section{PRIMARILY MECHANICALLY FUNCTIONAL TISSUES}

\section{Bone}

Bone is a mineralized collagen matrix responsible for primary load bearing in the musculoskeletal system. In addition, bone serves as an anchorage point for ligaments, tendons, and muscles to facilitate movement. Clinical applications of tissue-engineered bone often relate to the repair of critical-sized defects that cannot heal on their own. The primary objective of tis- 
Table 1. Advantages of scaffold-free techniques

\begin{tabular}{lc}
\hline Target tissues & Examples of commonly reported parameters \\
\hline Primarily mechanical function (e.g., musculoskeletal, cardiovascular) \\
Bone & Compressive and tensile stiffness, strength \\
Articular cartilage & Compressive and tensile stiffness, strength \\
Tendon, ligament & Tensile stiffness, strength, maximum force \\
Heart & Electrical conductance, contractility \\
Vessel & Burst pressure, recapitulation of layered structures \\
Nonmechanical tissues (e.g., metabolic, corneal) & \\
Liver & Albumin, $\alpha-1$ antitrypsin, P450 cytochrome \\
Cornea & Transparency, refractive power \\
\hline
\end{tabular}

sue engineers, therefore, is to engineer bone with compressive and tensile stiffness and strength reminiscent of native bone. Additionally, indicators of vascularization and mineralization are used to assess bone quality. Scaffoldfree approaches used in bone engineering are primarily cell-sheet-based, with few using aggregate culture.

Osteogenic cell sheets are used to generate tissue-engineered bone, targeting the compressive and mineral properties of native bone. Osteogenically differentiated MSC sheets rolled into cylindrical constructs showed mineralization and a compressive strength of $\sim 1.6 \mathrm{MPa}$ (Ma et al. 2010). The cell-sheet structure can be placed into critical-sized defects to facilitate new tissue formation, although additional validation of the mechanical properties of the newly formed bone is needed (Akahane et al. 2010). In vivo implantation promoted expression of bone genetic markers (i.e., collagen type I, osteocalcin, and osterix) in cell-sheet cocultures of osteogenic stromal cells and endothelial cells as compared with monocultures of osteogenic cells (Pirraco et al. 2014). Although bone-tissue engineering with scaffold-free cell sheets has been attempted, most of these studies do not assess the mechanical properties of formed tissues. As such, additional work analyzing the functional mechanical properties of cell-sheetbased, in vitro tissue-engineered bone is needed.

Few studies examine aggregate or spheroid culture for engineering large bone-tissue constructs, as aggregate studies are primarily used for differentiating progenitor cells into osteo- genic cells for future application in larger scaffold-free or scaffold-based techniques. Various scaffold-free aggregate techniques are able to generate mineralizing spheroids, but larger constructs are not often formed in subsequent steps (Hildebrandt et al. 2011). Studies using scaffold-free processes from cellular differentiation to large construct formation would improve our understanding of the potential applicability of scaffold-free techniques in bone-tissue engineering.

\section{Cartilage}

Articular cartilage is a load-bearing tissue that also serves to facilitate the smooth translation of diarthrodial joints. Unlike bone, however, articular cartilage does not heal itself; clinical application of tissue-engineered articular cartilage, then, serves to replace degenerated cartilage tissues. The primary benchmarks of articular cartilage tissue engineering are sufficient compressive and tensile properties. In addition, lubrication properties and integration ability are desirable. Scaffold-free methods, including the self-assembling process, cell-sheet engineering, and aggregate culture, have been applied in articular cartilage tissue engineering toward achieving the mechanical properties of native tissues.

The self-assembling process as a scaffoldfree tissue-formation technique has been extensively studied ( $\mathrm{Hu}$ and Athanasiou 2006) for engineering of articular cartilage. With this scaffold-free process, primary articular chon- 
J.K. Lee et al.

drocytes treated with matrix cross-linking enzyme lysyl oxidase-like protein 2 (LOXL2) can generate tissues achieving compressive and tensile stiffnesses up to $220 \mathrm{kPa}$ and $2.3 \mathrm{MPa}$, respectively (Makris et al. 2014). Using combinations of bioactive stimuli (i.e., TGF- $\beta 1$, chondroitinase-ABC) and/or mechanical stimuli (i.e., hydrostatic pressure), tissues with tensile stiffnesses up to 6.3 $\mathrm{MPa}$ and 2.1 $\mathrm{MPa}$ can be generated from passaged leporine and porcine cells, respectively (Murphy et al. 2013b; Arzi et al. 2015). Self-assembled articular cartilage derived from primary chondrocytes cultured in chondrogenic-inducing medium expressed superficial zone protein, imparting the tissue with lubrication capacity and frictional properties approaching those of native articular cartilage (Peng et al. 2014). The self-assembling process, thus, can be used to engineer native tissue-like neocartilage. Future work investigating the implantation of these lubricated and mechanically robust tissues in an orthotopic location would lead the field in generating functional tissue-engineered cartilage.

Other scaffold-free methods for cartilage tissue engineering include cell-sheet engineering and aggregate culture. In a technique similar to self-assembly, chondrocytes placed in a nonadherent well self-aggregate into "cartilage tissue analogs" expressing collagen type II and native tissue levels of glycosaminoglycan (Kraft et al. 2011). Moreover, these tissues were reported to have an equilibrium compressive Young's modulus on par with native tissue (Mohanraj et al. 2014). Cell-sheet engineering has also been used to engineer cartilage tissues; contraction of an MSC-derived cell sheet led to a tensile strength of $\sim 1.2 \mathrm{MPa}$ (Ando et al. 2008). Scaffold-free aggregate and cell-sheet culture methods are able to generate cartilage tissues that are mechanically viable. Additional investigation using these methods to achieve native tissue-like mechanical properties as well as lubrication would benefit efforts to engineer scaffold-free cartilage.

\section{Ligaments and Tendons}

Ruptured ligaments and tendons are often repaired with autologous or cadaver-derived grafts, which are limited in supply. Tissue engineering of ligaments and tendons thus aims to fill this need for mechanically robust replacements. Engineered ligaments and tendons are commonly assessed for various tensile testing criteria, such as tensile stiffness, strength, and force. Scaffold-free methods used in forming these tissues are akin to cell-sheet engineering, whereas spheroids have been used to a limited extent.

Cell-sheet methods in ligament and tendon engineering rely on the strong contractile forces of seeded cells. Monolayers of stromal cells cultured on laminin-coated substrates detached and organized into rod-like tissues anchored by silk sutures; these tissues reached a tensile force of $0.26 \mathrm{~N}$, a tangent modulus up to 15.4 MPa, and a tensile stress of $2.11 \mathrm{MPa}$ (Hairfield-Stein et al. 2007). Rolling of a tenocyte-derived cell sheet stimulated with ascorbic acid and connective tissue growth factor achieved a reported tensile stiffness of $\sim 200 \mathrm{~N} / \mathrm{mm}^{2}$ (MPa) and strongly expressed collagen type I and tenomodulin ( $\mathrm{Ni}$ et al. 2013). Additional work exploring layering or bundling of scaffold-free ligaments and tendons to achieve mechanically robust tissues with larger geometries would move the field closer to a replacement ligament or tendon.

The use of spheroid culture in tendon and ligament engineering is limited primarily to differentiation and phenotypic maintenance of cells for seeding on woven scaffolds. Scaffoldfree spheroids derived from anterior cruciate ligament cells became smaller over time, but increasingly expressed collagen and tenascin C and could colonize scaffolds (Hoyer et al. 2015). Hanging-drop spheroid culture of tenocytes similarly enhanced expression of tendon-associated genes (e.g., collagen type III, scleraxis) as compared with monolayer cultures, with ascorbic acid, insulin, and TGF- $\beta 1$ achieving higher expression levels than TGF- $\beta 1$ and insulin-like growth factor 1 (IGF-1) use (Theiss et al. 2015). Although the use of spheroids in tendon and ligament engineering shows the ability of scaffold-free culture to enhance relevant gene expression, these studies did not examine the functional mechanical properties of engineered 
Self-Assembly and Tissue Engineering

tissues. As such, additional work exploring the mechanical properties achievable through a combination of scaffold-free and scaffold-based methods for tendon and ligament engineering would benefit the field.

\section{Cardiac}

Cardiac tissues function primarily in contraction, relying on rapid electrical conductance to synchronize the heartbeat. In cases of myocardial infarction, large portions of the heart are damaged and cannot properly conduct these electrical signals. Cardiac tissue engineering aims to repair and replace damaged tissue to restore electrical conductivity and contractility toward reestablishing normal heart function. Cell-sheet engineering as a scaffold-free method dominates cardiac tissue engineering and aims primarily to achieve electrical conductance for synchronous contractility. More recently, efforts to vascularize engineered cardiac tissues before implantation have emerged.

Cell-sheet engineering techniques have been developed to form 3D cardiac tissues capable of electrical communication. Synchronous and spontaneous beating was achieved by layering cardiac cell sheets derived from embryonic cardiomyocytes (Shimizu et al. 2002). Electrical connectivity via the formation of gap junctions, as indicated by connexin 43 staining, has been observed in layered cardiac tissues (Shimizu et al. 2006; Matsuura et al. 2011). Because of the metabolic requirements of cardiac cells, vascularization of layered cardiac sheets is important for in vivo survival of the graft (Dilley and Morrison 2014). Multistep transplantation of ten three-layer cardiac sheets cocultured with endothelial cells promoted vascularization in vivo, resulting in a fused, 30-layer-thick cardiac tissue beating simultaneously (Shimizu et al. 2006). Electrical conductivity and synchronous beating in engineered cardiac tissues can thus be achieved with cell-sheet engineering. These studies show that in vivo tissue engineering of cell sheets induces vascularization. Additional studies exploring in vitro vascularization and in vivo integration of vascular cardiac tissues are needed to promote repair of damaged heart tissues.

\section{Vascular}

Diseases affecting the vascular system can lead to myocardial infarction, stroke, or peripheral limb ischemia. Vascular tissue engineering aims to replace segments of diseased vessels. To recapitulate native tissue function, engineered vessels should be able to withstand physiological burst pressures; additionally, they should resist cyclic loading fatigue and maintain an endothelium layer (Seifu et al. 2013). In the last two decades, vascular tissue engineering has diversified to include scaffold-free systems that include self-assembly, cell-sheet, and spheroidbased techniques.

The self-assembling process has seen limited application in vascular tissue engineering. Vascular rings were formed through self-assembly of smooth muscle cells before they were placed sequentially on a silicone mandrel and underwent fusion (self-organization) (Gwyther et al. 2011). Although the tensile mechanical properties of individual vascular rings were assessed (ultimate tensile strength of $100-500 \mathrm{kPa}$ ), the functional properties of the fused tubular structure were not. In addition to examining burst pressure, future work using both self-assembly and self-organization could control cell placement (e.g., endothelial cells at the vascular ring center, smooth muscle cells in the media layer, and fibroblasts at the outermost edge) to create a tissue-engineered vessel with structural morphology and mechanical properties reminiscent of vascular tissues.

Vascular tissue formation using cell-sheet engineering is the most popular scaffold-free system used. Sequential sheets of human vascular smooth muscle cells and fibroblasts wrapped around a mandrel fused to form a tubular vessel capable of endothelialization and showed a reported "burst strength" $>2000 \mathrm{mmHg}(\sim 265 \mathrm{kPa})$ (L'Heureux et al. 1998). Subsequent iterations of this method produced fibroblast- and endothelial-cell-based vessels with reported burst pressures of more than $3500 \mathrm{mmHg} \quad(\sim 465 \mathrm{kPa}) \quad$ (L'Heureux et al. 2006). Ascorbic acid-treated MSCs can also be used in a similar process to generate a 
J.K. Lee et al.

cell-sheet-based vascular tissue with suitable suture loading strength (Zhao et al. 2012). Finally, cell-sheet methods can be combined with scaffold-based technologies to enhance functional properties. Primary smooth musclecell-derived sheets seeded onto electrospun collagen/poly $(\varepsilon$-caprolactone $)$ scaffolds achieved additional increases in tensile strength of vessels compared with the scaffold alone (Ahn et al. 2015). These increases in tensile properties are encouraging, although burst pressure was not assessed in this study (Ahn et al. 2015). These studies show that cell-sheet-based vascular tissue engineering can achieve functional burst pressure properties exceeding those of native tissue. Toward potentially engineering contractile arterial vessels, future work should include smooth muscle cell phenotypic maintenance and/or differentiation and their incorporation into a mechanically robust vascular graft.

Finally, a combination of scaffold-free spheroid formation and the self-organizing technique of bioprinting has been used to a limited extent in vascular tissue engineering. This process used agarose as a mold to support the build process in bioprinting spheroids (formed via pellet culture) composed of smooth muscle cells and fibroblasts (Norotte et al. 2009). Layer-by-layer composition in this study allowed for the design of a double-layered vascular wall showing patterns of smooth muscle cell and fibroblast organization (Norotte et al. 2009). Interestingly, placement of stemcell-based spheroids on a prestretched electrospun scaffold resulted in incomplete fusion and hole formation in tissue-engineered vessels, suggesting that the scaffold may impede fusion (Beachley et al. 2014). Although burst pressure as a functional parameter was not assessed in these spheroid-based studies, the results show the ability to finely control structural architecture in vascular tissue engineering and achieve small-diameter vessels $(<5 \mathrm{~mm})$. Additional work to enhance fusion of spheroid structures and produce mechanically viable vessels, toward achieving branching vasculature capable of withstanding burst pressures, would greatly benefit the field.

\section{NONMECHANICAL TISSUES}

Liver

Liver diseases including fibrosis and viral infections have driven the need for alternative sources of healthy liver tissue because donors are limited. The most successful option for complete liver failure remains liver transplantation. Primary hepatocyte transplantation involving injection of a cell suspension has been used, but transplantation of engineered tissues is still under development. Liver tissue engineering is meant to both maintain hepatocyte phenotype in culture and to differentiate progenitor cells into mature hepatocytes. To create a functional tissue-engineered solution, engineers focus on protein and metabolite secretion, primarily production of albumin, $\alpha 1$ antitrypsin (A1AT), and the P450 cytochrome enzyme. Scaffold-free methods of aggregate or spheroid culture and cell-sheet techniques have been used to achieve these objectives.

Scaffold-free spheroids are formed from a variety of cell sources and are the primary scaffold-free method used in liver tissue engineering, as spheroids provide a means to maintain the phenotype of liver cells. The dimensions of pelleted aggregates can influence both the immediate and long-term expression levels of liver-specific albumin (Gevaert et al. 2014) and should be considered in liver-tissue engineering. Additionally, hepatocyte spheroids have been shown to survive and maintain their phenotype at least 3 days when implanted in vivo (Ota et al. 1997). Coculture of hepatocytes and other cell types can further enhance the phenotypic maintenance of hepatocytes. For instance, cocultures of hepatocytes and hepatic stellate cells induced increased expression of albumin and cytochrome P450 compared with hepatocytes alone (Wong et al. 2011). Similarly, aggregates formed via coculture of hepatocytes and pancreatic islet cells not only maintained hepatocytic (and pancreatic) phenotypes but enhanced expression of liver-specific proteins over hepatocyte aggregates (Jun et al. 2013). These studies thus show the importance of scaffold-free spheroid culture not only to maintain but also to enhance hepatocyte phenotype, es- 
pecially when hepatocytes are cultured with support cells. Future work should validate the long-term phenotypic stability of hepatocytes cultured as spheroids and their potential in long-term restoration of liver function when implanted in vivo.

Cell-sheet culture in liver-tissue engineering involves coculture of hepatocytes with an additional cell source or layering of multiple hepatocyte sheets. Hepatocyte cell sheets showed robust expression of albumin as evaluated via immunohistochemistry; increases in protein production correlated with enhanced liver-tissue volume as a result of layering multiple cell sheets (Ohashi et al. 2007). In addition to robust albumin and A1AT production, cell sheets derived from hepatocytes cocultured with fibroblasts promoted enhanced vascularization after subcutaneous implantation when compared with hepatocyte-derived sheets (Sakai et al. 2015). This scaffold-free coculture technique could address the pressing need for vascularization after transplantation to ensure survival of engineered liver tissues. It is important to note that the in vivo vascularization of engineered liver tissues is encompassed within the in vivo tissue-engineering methodology; successful in vitro engineering of liver vasculature would greatly advance the field. Scaffold-free cell sheets of hepatocytes alone or in coculture are thus able to express proteins indicative of liver function and induce vascularization of implanted tissues.

\section{Cornea}

The cornea is a transparent and avascular ocular structure that provides physical protection to the eye and serves as an optical interface. An epithelium and endothelium layer comprise a combined $\sim 10 \%$ of the corneal thickness and act primarily as a barrier and integration point to the remainder of the eye, respectively. Structurally, aligned collagen fibrils, termed lamellae, comprise the bulk of the corneal stroma, which represents $90 \%$ of corneal thickness (Ghezzi et al.2015). Proteoglycans between lamellae layers contribute to corneal transparency, a unique attribute of this tissue. Diseased or damaged corneal tissues can lead to vision loss and blindness, creating a clinical need for tissue-engineered corneas for transplantation. The primary challenge of engineering the cornea is to create a transparent structure with suitable matrix organization that confers substantial refractive power and mechanical protection (Ghezzi et al. 2015). Full-thickness corneal tissue engineering has primarily been completed with scaffold-based methods, although cell-sheet systems have been used to engineer certain layers.

Cell-sheet engineering is the predominant scaffold-free method studied for corneal regeneration. Researchers created autologous epithelial cell sheets that were able to restore the cornea's transparency and natural barrier function and improve visual acuity in human patients (Nishida et al. 2004b); this technique was previously shown in a rabbit corneal model (Nishida et al. 2004a). Although these studies successfully created an epithelial cell sheet, they did not create the stroma. Other cell-sheet work attempted to create thicker corneal tissues by altering the cell-sheet growth substrate (Teichmann et al. 2013) or by engineering specifically the endothelium layer (Madathil et al. 2014). Despite this exciting work in stroma or endothelium engineering, these studies have not yet examined the functional parameters of transparency and physical protection. Cell sheets are promising for the creation of transparent and protective corneal layers. Additional studies combining various cell-sheet layers may elucidate the potential of generating full-thickness corneal transplants.

\section{CONCLUSIONS AND FUTURE DIRECTIONS}

In the last few decades, the tissue-engineering field has made tremendous strides toward creating functional tissues able to replace those damaged by disease, trauma, or age. Scaffoldfree tissue engineering recently emerged as an alternative approach that uses only cells and signals, aiming to exploit the benefits of scaffoldfree systems. Within scaffold-free systems, selforganization and self-assembling processes can be defined based on whether external energy is applied (Fig. 1). As the field progresses in the 
J.K. Lee et al.

continued use of scaffold-free systems, it will become critical to create stricter definitions for terminology used to denote various techniques; this issue is particularly important in the development of spheroid, aggregate, and pellet-based technologies, terms often used interchangeably. Depending on the target tissue of interest, a given scaffold-free (or even scaffoldbased) method may be preferable. This review summarizes the current progress in the tissueengineering field, focusing primarily on scaffold-free techniques. Specifically, we highlight the recent advances and existing limitations in biomimetic in vitro tissue engineering, toward creating tissues that truly restore the function of the intended tissue targets. Although the field has seen expansive growth with the advent of new technologies, this review highlights remaining hurdles that need to be addressed for clinical translation.

Extensive progress in tissue engineering has resulted in tissues that recapitulate certain metrics of target tissues; to assess the long-term functionality and facilitate clinical translation, however, increased development and standardization of appropriate animal models are needed. These tissue-engineering models should match not only the disease characteristic, but the defect characteristics as well. For example, osteoarthritis models should aim to better recapitulate not only the inflammatory environment, but also the size and shape of a cartilage defect. In addition to appropriately modeling the disease state, standardization of animal models across research groups would assist in direct comparisons of studies. Use of select FDA-approved models may guide animal selection. In scaffold-free systems, specifically, achievement of sufficient mechanical properties is critical to the survival of the implant within the host environment. In the case of articular cartilage replacement, until mechanically biomimetic tissues can be engineered using scaffold-free systems, it may be advisable to explore rehabilitation techniques that use unloading of the patient's joint until the neotissue has matured mechanically. Because of the dependence on high numbers of cells, scaffold-free tissueengineered constructs may need to address the permanence of these cells within the construct to determine that cells do not leave the implanted neotissue and elicit adverse effects elsewhere in the host. Continued improvements to animal models will facilitate the translation of engineered tissues to human patients. Until truly biomimetic tissue is created, use of scaffoldfree techniques in the clinic may progress with appropriate rehabilitation and postoperative procedures in place to ensure neotissue maturation and development.

Cell sourcing is arguably the most limiting step of tissue engineering-both scaffolds and signals can be synthetically created, whereas cells must be derived from a natural source and are thus a limited resource. The high cell numbers needed for scaffold-free techniques render them particularly vulnerable to cellsourcing issues. Using aggregate culture protocols, cell-sourcing limitations can be addressed, as these approaches can be an effective means to promote the desired cell phenotype in a 3D environment. Because primary cells are limited in availability, most tissue engineers select progenitor cells that can be differentiated using aggregate culture. Once the desired phenotype has been obtained, these cells can be used in a subsequent scaffold-free or scaffold-based method to generate constructs of clinically relevant dimensions. Increased development of our ability to differentiate progenitor cells or maintain primary cells in $3 \mathrm{D}$ culture can ultimately address the significant cell numbers needed in scaffoldfree in vitro biomimetic tissue engineering.

Although this review focuses on scaffoldfree systems, continued progress in tissue engineering may require the simultaneous use of scaffold-free and scaffold-based techniques as the complexity of engineered tissues increases. As mentioned previously, many cells are anchorage-dependent and are not suitable for use in scaffold-free systems. For instance, osteoblasts require a scaffold for their survival. Therefore, to form a biphasic osteochondral graft, a cell-laden bone scaffold may be used in conjunction with scaffold-free neocartilage. Identification of the appropriate system-scaffold-free or scaffold-based-for independent cell types will further the field in developing 
more complex tissues through the combination of various systems.

Finally, enhanced understanding of the development of various tissue types will aid in identification of whether scaffold-free or scaffold-based systems are most appropriate. In this article, we highlight the parallels between scaffold-free neocartilage generation and in vivo development of articular cartilage (Fig. 2). Mesenchymal condensation of cartilaginous precursors occurs in the absence of a scaffold; tissue engineering with chondrocytes in a scaffoldfree process thus reflects the developmental environment. The work in developmental biology can thus inform the selection of tissue-engineering modalities. Increased understanding of the processes by which various tissues and organs develop will aid the selection of scaffold-free or scaffold-based techniques.

A key focus of this work is the self-assembling process, which results in functional tissue formation in a cell-driven manner that requires no external input of energy. In some tissue types (i.e., cartilage), the self-assembling process mimics natural mechanisms of developmental biology. By studying the self-assembling platform, enhanced understanding of development may be achieved. Conversely, our current understanding of developmental processes may be applied to self-assembling techniques, toward discovering methods to enhance the functional properties of neotissue. Ultimately, engineered neotissue will reach a level of complexity recapitulating native tissue functions, allowing neotissue not just to repair, but also to regenerate diseased tissues.

\section{REFERENCES}

Adkisson HD, Gillis MP, Davis EC, Maloney W, Hruska KA. 2001. In vitro generation of scaffold independent neocartilage. Clin Orthop Relat Res 391: S280-S294.

Ahn H, Ju YM, Takahashi H, Williams DF, Yoo JJ, Lee SJ, Okano T, Atala A. 2015. Engineered small diameter vascular grafts by combining cell sheet engineering and electrospinning technology. Acta Biomater 16: $14-22$.

Akahane M, Shigematsu H, Tadokoro M, Ueha T, Matsumoto T, Tohma Y, Kido A, Imamura T, Tanaka Y. 2010 Scaffold-free cell sheet injection results in bone formation. J Tissue Eng Regen Med 4: 404-411.
Amini AR, Laurencin CT, Nukavarapu SP. 2012. Bone tissue engineering: Recent advances and challenges. Crit Rev Biomed Eng 40: 363-408.

Ando W, Tateishi K, Katakai D, Hart DA, Higuchi C, Nakata K, Hashimoto J, Fujie H, Shino K, Yoshikawa H, et al. 2008. In vitro generation of a scaffold-free tissue-engineered construct (TEC) derived from human synovial mesenchymal stem cells: Biological and mechanical properties and further chondrogenic potential. Tissue Eng Part A 14: 2041-2049.

Arzi B, DuRaine GD, Lee CA, Huey DJ, Borjesson DL, Murphy BG, Hu JC, Baumgarth N, Athanasiou KA. 2015. Cartilage immunoprivilege depends on donor source and lesion location. Acta Biomater 23: 72-81.

Athanasiou KA, Eswaramoorthy R, Hadidi P, Hu JC. 2013. Self-organization and the self-assembling process in tissue engineering. Аnnu Rev Biomed Eng 15: 115-136.

Athanasiou KA, Responte DJ, Brown WE, Hu JC. 2015. Harnessing biomechanics to develop cartilage regeneration strategies. J Biomech Eng 137: 020901.

Athens AA, Makris EA, Hu JC. 2013. Induced collagen cross-links enhance cartilage integration. PLoS ONE 8: e60719.

Avula MN, Rao AN, McGill LD, Grainger DW, Solzbacher F. 2014. Foreign body response to subcutaneous biomaterial implants in a mast cell-deficient $\mathrm{Kit}^{w-S h}$ murine model. Acta Biomater 10: 1856-1863.

Beachley V, Kasyanov V, Nagy-Mehesz A, Norris R, Ozolanta I, Kalejs M, Stradins P, Baptista L, da Silva K, Grainjero J, et al. 2014. The fusion of tissue spheroids attached to prestretched electrospun polyurethane scaffolds. J Tissue Eng 5: $1-11$.

Bentley G, Biant LC, Vijayan S, Macmull S, Skinner JA, Carrington RWJ. 2012. Minimum ten-year results of a prospective randomised study of autologous chondrocyte implantation versus mosaicplasty for symptomatic articular cartilage lesions of the knee. J Bone Joint Sur Br 94: 504-509.

Bhumiratana S, Eton RE, Oungoulian SR, Wan LQ, Ateshian GA, Vunjak-Novakovic G. 2014. Large, stratified, and mechanically functional human cartilage grown in vitro by mesenchymal condensation. Proc Natl Acad Sci 11: 6940-6945.

Brittberg M, Lindahl A, Nilsson A, Ohlsson C, Isaksson O, Peterson L. 1994. Treatment of deep cartilage defects in the knee with autologous chondrocyte transplantation. $N$ Engl J Med 331: 889-895.

Brodland GW. 2002. The differential interfacial tension hypothesis (DITH): A comprehensive theory for the selfrearrangement of embryonic cells and tissues. J Biomech Eng 124: 188-197.

Chaparro FJ, Matusicky ME, Allen MJ, Lannutti JJ. 2015. Biomimetic microstructural reorganization during suture retention strength evaluation of electrospun vascular scaffolds. J Biomed Mater Res B Appl Biomater 104: 15251534.

Darling EM, Athanasiou KA. 2005. Rapid phenotypic changes in passaged articular chondrocyte subpopulations. J Orthop Res 23: 425-432.

DeLise AM, Fischer L, Tuan RS. 2000. Cellular interactions and signaling in cartilage development. Osteoarthritis Cartilage 8: 309-334. 
J.K. Lee et al.

Diekman BO, Christoforou N, Willard VP, Sun H, SanchezAdams J, Leong KW, Guilak F. 2012. Cartilage tissue engineering using differentiated and purified induced pluripotent stem cells. Proc Natl Acad Sci 109: 19172-19177.

Dilley RJ, Morrison WA. 2014. Vascularisation to improve translational potential of tissue engineering systems for cardiac repair. Int J Biochem Cell Biol 56: 38-46.

DuRaine GD, Brown WE, Hu JC, Athanasiou KA. 2015. Emergence of scaffold-free approaches for tissue engineering musculoskeletal cartilages. Ann Biomed Eng 43: 543-554.

Gevaert E, Dollé L, Billiet T, Dubruel P, van Grunsven L, van Apeldoorn A, Cornelissen R. 2014. High throughput micro-well generation of hepatocyte micro-aggregates for tissue engineering. PLoS ONE 9: e105171.

Ghezzi CE, Rnjak-Kovacina J, Kaplan DL. 2015. Corneal tissue engineering: Recent advances and future perspectives. Tissue Eng Part B Rev 21: 278-287.

Gurkan UA, Kishore V, Condon KW, Bellido TM, Akkus O. 2011. A scaffold-free multicellular three-dimensional in vitro model of osteogenesis. Calcif Tissue Int 88: 388401.

Gwyther TA, Hu JZ, Billiar KL, Rolle MW. 2011. Directed cellular self-assembly to fabricate cell-derived tissue rings for biomechanical analysis and tissue engineering. J Vis Exp 57: e3366.

Hairfield-Stein M, England C, Paek HJ, Gilbraith KB, Dennis R, Boland E, Kosnik P. 2007. Development of selfassembled, tissue-engineered ligament from bone marrow stromal cells. Tissue Eng 13: 703-710.

Hildebrandt C, Büth H, Thielecke H. 2011. A scaffold-free in vitro model for osteogenesis of human mesenchymal stem cells. Tissue Cell 43: 91-100.

Hoyer M, Meier C, Breier A, Hahner J, Heinrich G, Drechsel N, Meyer M, Rentsch C, Garbe LA, Ertel W, et al. 2015. In vitro characterization of self-assembled anterior cruciate ligament cell spheroids for ligament tissue engineering. Histochem Cell Biol 143: 289-300.

Hu JC, Athanasiou KA. 2006. A self-assembling process in articular cartilage tissue engineering. Tissue Eng 12: 969979.

Huey DJ, Hu JC, Athanasiou KA. 2012. Unlike bone, cartilage regeneration remains elusive. Science 338: 917-921.

Jun Y, Kang AR, Lee JS, Jeong GS, Ju J, Lee DY, Lee SH. 2013. $3 \mathrm{D}$ co-culturing model of primary pancreatic islets and hepatocytes in hybrid spheroid to overcome pancreatic cell shortage. Biomaterials 34: 3784-3794.

Kraft JJ, Jeong C, Novotny JE, Seacrist T, Chan G, Domzalski M, Turka CM, Richardson DW, Dodge GR. 2011. Effects of hydrostatic loading on a self-aggregating, suspension culture-derived cartilage tissue analog. Cartilage 2: 254264.

Krieg M, Arboleda-Estudillo Y, Puech PH, Käfer J, Graner F, Müller DJ, Heisenberg CP. 2008. Tensile forces govern germ-layer organization in zebrafish. Nat Cell Biol 10: 429-436.

L'Heureux N, Pâquet S, Labbé R, Germain L, Auger FA. 1998. A completely biological tissue-engineered human blood vessel. FASEB J 12: 47-56.

L'Heureux N, Dusserre N, Konig G, Victor B, Keire P, Wight TN, Chronos NA, Kyles AE, Gregory CR, Hoyt G, et al.
2006. Human tissue-engineered blood vessels for adult arterial revascularization. Nat Med 12: 361-365.

Lim HC, Bae JH, Song SH, Park YE, Kim SJ. 2012. Current treatments of isolated articular cartilage lesions of the knee achieve similar outcomes. Clin Orthop Relat Res 470: 2261-2267.

Lu Y, Adkisson HD, Bogdanske J, Kalscheur V, Maloney W, Cheung R, Grodzinsky AJ, Hruska KA, Markel MD. 2005. In vivo transplantation of neonatal ovine neocartilage allografts: Determining the effectiveness of tissue transglutaminase. J Knee Surg 18: 31-42.

Ma D, Ren L, Liu Y, Chen F, Zhang J, Xue Z, Mao T. 2010. Engineering scaffold-free bone tissue using bone marrow stromal cell sheets. J Orthop Res 28: 697-702.

Madathil BK, Kumar PRA, Kumary TV. 2014. N-isopropylacrylamide-co-glycidylmethacrylate as a thermoresponsive substrate for corneal endothelial cell sheet engineering. Biomed Res Int doi: 10.1155/2014/450672.

Makris EA, Responte DJ, Paschos NK, Hu JC, Athanasiou KA. 2014. Developing functional musculoskeletal tissues through hypoxia and lysyl oxidase-induced collagen cross-linking. Proc Natl Acad Sci 111: E4832-E4841.

Makris EA, Gomoll AH, Malizos KN, Hu JC, Athanasiou KA. 2015. Repair and tissue engineering techniques for articular cartilage. Nat Rev Rheumatol 11: 21-34.

Manning ML, Foty RA, Steinberg MS, Schoetz EM. 2010. Coaction of intercellular adhesion and cortical tension specifies tissue surface tension. Proc Natl Acad Sci 107: $12517-12522$.

Matsuura K, Masuda S, Haraguchi Y, Yasuda N, Shimizu T, Hagiwara N, Zandstra PW, Okano T. 2011. Creation of mouse embryonic stem cell-derived cardiac cell sheets. Biomaterials 32: 7355-7362.

McAllister TN, Maruszewski M, Garrido SA, Wystrychowski W, Dusserre N, Marini A, Zagalski K, Fiorillo A, Avila H, Manglano X, et al. 2009. Effectiveness of haemodialysis access with an autologous tissue-engineered vascular graft: A multicentre cohort study. Lancet 373: 14401446.

McCormick F, Cole BJ, Nwachukwu B, Harris JD, Adkisson Iv HD, Farr J. 2013. Treatment of focal cartilage defects with a juvenile allogeneic 3-dimensional articular cartilage graft. Oper Tech Sports Med 21: 95-99.

Mohanraj B, Farran AJ, Mauck RL, Dodge GR. 2014. Timedependent functional maturation of scaffold-free cartilage tissue analogs. J Biomech 47: 2137-2142.

Murphy MK, Masters TE, Hu JC, Athanasiou KA. 2013a. Engineering a fibrocartilage spectrum through modulation of aggregate redifferentiation. Cell Transplant 24: 235-245.

Murphy MK, DuRaine GD, Reddi A, Hu JC, Athanasiou KA. 2013b. Inducing articular cartilage phenotype in costochondral cells. Arthritis Res Ther 15: R214.

Murphy MK, Huey DJ, Hu JC, Athanasiou KA. 2015. TGF$\beta 1$, GDF-5, and BMP-2 stimulation induces chondrogenesis in expanded human articular chondrocytes and marrow-derived stromal cells. Stem Cells 33: 762-773.

Ni M, Rui YF, Tan Q, Liu Y, Xu LL, Chan KM, Wang Y, Li G. 2013. Engineered scaffold-free tendon tissue produced by tendon-derived stem cells. Biomaterials 34: 2024-2037. 
Nishida K, Yamato M, Hayashida Y, Watanabe K, Maeda N, Watanabe H, Yamamoto K, Nagai S, Kikuchi A, Tano Y, et al. 2004a. Functional bioengineered corneal epithelial sheet grafts from corneal stem cells expanded ex vivo on a temperature-responsive cell culture surface. Transplantation 77: 379-385.

Nishida K, Yamato M, Hayashida Y, Watanabe K, Yamamoto K, Adachi E, Nagai S, Kikuchi A, Maeda N, Watanabe H, et al. 2004b. Corneal reconstruction with tissue-engineered cell sheets composed of autologous oral mucosal epithelium. N Engl J Med 351: 1187-1196.

Norotte C, Marga FS, Niklason LE, Forgacs G. 2009. Scaffold-free vascular tissue engineering using bioprinting. Biomaterials 30: 5910-5917.

Ofek G, Revell CM, Hu JC, Allison DD, Grande-Allen KJ, Athanasiou KA. 2008. Matrix development in self-assembly of articular cartilage. PLoS ONE 3: e2795.

Ohashi K, Yokoyama T, Yamato M, Kuge H, Kanehiro H, Tsutsumi M, Amanuma T, Iwata $\mathrm{H}$, Yang J, Okano T, et al. 2007. Engineering functional two- and three-dimensional liver systems in vivo using hepatic tissue sheets. Nat Med 13: 880-885.

Ong SY, Dai H, Leong KW. 2006. Inducing hepatic differentiation of human mesenchymal stem cells in pellet culture. Biomaterials 27: 4087-4097.

Ota K, Saito S, Fujisawa K, Tanaka N. 1997. Xenotransplantation of spheroidal aggregate-cultured hepatocytes. Transplant Proc 29: 912-913.

Peng G, McNary SM, Athanasiou KA, Reddi AH. 2014. Surface zone articular chondrocytes modulate the bulk and surface mechanical properties of the tissue engineered cartilage. Tissue Eng Part A 20: 3332-3341.

Pirraco RP, Iwata T, Yoshida T, Marques AP, Yamato M, Reis RL, Okano T. 2014. Endothelial cells enhance the in vivo bone-forming ability of osteogenic cell sheets. Lab Invest 94: 663-673.

Raghothaman D, Leong MF, Lim TC, Toh JK, Wan AC, Yang Z, Lee EH. 2014. Engineering cell matrix interactions in assembled polyelectrolyte fiber hydrogels for mesenchymal stem cell chondrogenesis. Biomaterials 35: 2607 2616.

Sakai Y, Yamanouchi K, Ohashi K, Koike M, Utoh R, Hasegawa $\mathrm{H}$, Muraoka I, Suematsu T, Soyama A, Hidaka M, et al. 2015. Vascularized subcutaneous human liver tissue from engineered hepatocyte/fibroblast sheets in mice. Biomaterials 65: $66-75$.
Seifu DG, Purnama A, Mequanint K, Mantovani D. 2013. Small-diameter vascular tissue engineering. Nat Rev Cardiol 10: 410-421.

Shimizu T, Yamato M, Akutsu T, Shibata T, Isoi Y, Kikuchi A, Umezu M, Okano T. 2002. Electrically communicating three-dimensional cardiac tissue mimic fabricated by layered cultured cardiomyocyte sheets. J Biomed Mater Res 60: $110-117$

Shimizu T, Sekine H, Yang J, Isoi Y, Yamato M, Kikuchi A, Kobayashi E, Okano T. 2006. Polysurgery of cell sheet grafts overcomes diffusion limits to produce thick, vascularized myocardial tissues. FASEB J 20: 708-710.

Shimizu T, Akahane M, Morita Y, Omokawa S, Nakano K, Kira T, Onishi T, Inagaki Y, Okuda A, Kawate K, et al. 2015. The regeneration and augmentation of bone with injectable osteogenic cell sheet in a rat critical fracture healing model. Injury 46: 1457-1464.

Tavella S, Raffo P, Tacchetti C, Cancedda R, Castagnola P. 1994. N-CAM and N-cadherin expression during in vitro chondrogenesis. Exp Cell Res 215: 354-362.

Teichmann J, Valtink M, Gramm S, Nitschke M, Werner C, Funk RH, Englemann K. 2013. Human corneal endothelial cell sheets for transplantation: Thermo-responsive cell culture carriers to meet cell-specific requirements. Acta Biomater 9: 5031-5039.

Theiss F, Mirsaidi A, Mhanna R, Kümmerle J, Glanz S, Bahrenberg G, Tiaden AN, Richards PJ. 2015. Use of biomimetic microtissue spheroids and specific growth factor supplementation to improve tenocyte differentiation and adaptation to a collagen-based scaffold in vitro. Biomaterials 69: 99-109.

Tuan RS. 2007. A second-generation autologous chondrocyte implantation approach to the treatment of focal articular cartilage defects. Arthritis Res Ther 9: 109.

Vunjak-Novakovic G, Martin I, Obradovic B, Treppo S, Grodzinsky AJ, Langer R, Freed LE. 1999. Bioreactor cultivation conditions modulate the composition and mechanical properties of tissue-engineered cartilage. J Orthop Res 17: 130-138.

Wong SF, No DY, Choi YY, Kim DS, Chung BG, Lee SH 2011. Concave microwell based size-controllable hepatosphere as a three-dimensional liver tissue model. Biomaterials 32: 8087-8096.

Zhao J, Liu L, Wei J, Ma D, Geng W, Yan X, Zhu J, Du H, Liu Y, Li L, et al. 2012. A novel strategy to engineer smalldiameter vascular grafts from marrow-derived mesenchymal stem cells. Artif Organs 36: 93-101. 


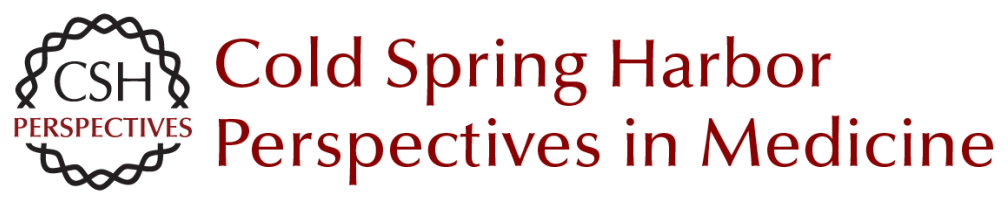

\section{The Self-Assembling Process and Applications in Tissue Engineering}

Jennifer K. Lee, Jarrett M. Link, Jerry C.Y. Hu and Kyriacos A. Athanasiou

Cold Spring Harb Perspect Med 2017; doi: 10.1101/cshperspect.a025668 originally published online March 27, 2017

\section{Subject Collection Tissue Engineering and Regenerative Medicine}

The Heart and Great Vessels

Ekene Onwuka, Nakesha King, Eric Heuer, et al.

Three-Dimensional Bioprinting Strategies for

Tissue Engineering

Yu Shrike Zhang, Rahmi Oklu, Mehmet Remzi Dokmeci, et al.

Honing Cell and Tissue Culture Conditions for Bone and Cartilage Tissue Engineering Johnny Lam, Esther J. Lee, Elisa C. Clark, et al.

Tissue Engineering Functional Gastrointestinal Regions: The Importance of Stem and Progenitor Cells

Andrew Trecartin and Tracy Grikscheit

\author{
Historical Perspective and Future Direction of \\ Blood Vessel Developments \\ Sashka Dimitrievska and Laura E. Niklason \\ Craniofacial Tissue Engineering \\ Weibo Zhang and Pamela Crotty Yelick
}

The Self-Assembling Process and Applications in Tissue Engineering Jennifer K. Lee, Jarrett M. Link, Jerry C.Y. Hu, et al.

Biologic Scaffolds

Alessandra Costa, Juan Diego Naranjo, Ricardo Londono, et al.

For additional articles in this collection, see http://perspectivesinmedicine.cshlp.org/cgi/collection/ 